\section{IL-2 COMBINATION WITH IMMTAC OVERCOMES CD163 + TAM-LIKE M2 MACROPHAGE INHIBITION OF IMMTAC-MEDIATED T CELL KILLING OF TUMOR CELLS}

Rahul Khanolkar*, Revashnee Naidoo, Esra Güç, Emma Leach, Sarah Stanhope, Duncan Gascoyne, Laura Collins, Koustubh Ranade, Adel Benlahrech. Immunocore, Abingdon, UK

Background ImmTAC molecules are bispecific fusion proteins consisting of an affinity-enhanced $\mathrm{T}$ cell receptor fused to an anti-CD3 effector that can redirect $\mathrm{T}$ cells to target cells. Tebentafusp, a gp100-directed ImmTAC, has demonstrated survival benefit in metastatic uveal melanoma [1]. We previously reported that a tumor microenvironment with a high immunosuppressive $\mathrm{CD} 163+$ tumor-associated macrophage (TAM): CD3 $\mathrm{T}$ cell ratio was associated with reduced benefit from tebentafusp [2]. Here, we explored whether IL-2 could potentiate $\mathrm{T}$ cells to overcome inhibition of ImmTAC-mediated killing by TAM-like M2 macrophages.

Methods Tumor biopsies from a Phase 2 trial of metastatic uveal melanoma HLA-A*02:01+ patients treated with tebentafusp (NCT02570308) were used to quantify CD163+ TAMs and $\mathrm{CD} 3+\mathrm{T}$ cells by immunohistochemistry $(\mathrm{N}=107)$ and to measure gene expression by bulk RNAseq $(\mathrm{N}=70)$. Pro-inflammatory M1 and TAM-like M2 macrophages were generated in vitro from healthy donors $(\mathrm{N}=5)$ and their effect on ImmTAC-mediated $\mathrm{T}$ cell activation and tumor killing was assessed against THP-1 tumor cells. T cells were untreated or pre-treated for 4 days with commercially sourced IL-2 or IL15.

Results In vitro, ImmTAC-mediated $\mathrm{T}$ cell killing of tumor cells was reduced by $85 \pm 5 \%$ in the presence of TAM-like M2 but not M1 macrophages. Consistent with this finding, below median CD163:CD3 ratio was associated with greater tumor shrinkage (TS) (odds ratio $\mathrm{OR}=2.9, \mathrm{p}=0.014$ ) and longer overall survival (OS) (hazard ratio $\mathrm{HR}=0.4, \mathrm{p}=0.001$ ) in tebentafusp-treated patients. We next explored in vitro whether the T cell activating cytokines IL-2 and IL-15 could overcome TAM-mediated inhibition of $\mathrm{T}$ cell redirection by ImmTAC. At clinically relevant doses, IL-2 but not IL-15 treatment resulted in dose dependent restoration of ImmTACmediated $\mathrm{T}$ cell killing of tumor cells in the presence of TAM-like M2 macrophages - $60 \%$ and $83 \%$ restoration of killing at 50 and $150 \mathrm{U} / \mathrm{ml}$ of IL-2, respectively. Consistent with this observation, increased expression of IL2RB (HR 0.3, $\mathrm{p}<0.001$ ) and IL2RG (HR 0.4, $\mathrm{p}=0.002$ ), but not IL2RA (HR $0.8, p=0.5)$ in tumors was associated with longer OS on tebentafusp.

Conclusions Low CD163+ TAM to CD3 T cell ratio and high IL2RB/G expression in tumors at baseline were associated with longer OS and greater TS in tebentafusp-treated metastatic uveal melanoma patients in a Phase 2 trial. In vitro, TAM-like M2 macrophages suppressed ImmTAC-mediated T cell killing of tumor cells, an effect abrogated by IL-2. These observations provide strong rationale for combining IL-2 biased to IL2RB/G with ImmTAC molecules to enhance benefit in tumors with high levels of TAMs.

Trial Registration NCT02570308

\section{REFERENCES}

1. Piperno-Neumann S, Hassel JC, Rutkowski $P$ et al. Abstract CT002: Phase 3 randomized trial comparing tebentafusp with investigator's choice in first line metastatic uveal melanoma. Cancer Res. 2021; 81 (13 Supplement) CT002.

2. Hassel JC, Benlahrech A, Stanhope S, et al. Abstract 1673: Uveal melanoma study patients with low CD163:CD3 ratio in tumor biopsy and low serum IL-6 showed enhanced tumor shrinkage (TS) and overall survival (OS) on tebentafusp. Cancer Res. 2021; 81 (13 Supplement) 1673.

Ethics Approval The Oxford A REC approved protocol 13/SC/ 0226 was used to obtain written consent for all blood donations and was fully approved by the National Research Ethics Committee South Central.

http://dx.doi.org/10.1136/jitc-2021-SITC2021.571 